\title{
Mobility Aware Co-operative MAC Protocol for Reliable Transmission in VANET
}

\author{
M. Manoj, A.Ch.Sudhir
}

\begin{abstract}
The Medium Access Control (MAC) etiquette is liable for partaking the communal medium amongst the competing nodules. The unique characteristics of VANET enforce many restrictions onto the MAC protocol design. The main aims of a VANET MAC protocol are fairness, Quality of Service (QoS), reliability and so on. In case of $\mathrm{V} 2 \mathrm{~V}$ communication in VANET, if the target vehicle is not reachable, then packets may not be transmitted correctly. In this paper, a Mobility Aware Cooperative MAC protocol (MAC-MAC) for successful transmission in VANET is proposed. In this protocol, when data is transmitted from $V 2 V$, the packet error rate (PER) and packet delivery ratio (PDR) metrics are checked at the receiver end. Depending on these values, the transmission mode of the source is changed as DIRECT or COOPERATIVE. In cooperative mode, the source selects the potential relay nodes based on the residence time and distance to the receiver. By experimental results, it is shown that the proposed MAC-MAC protocol has reduced packet drops, delay and overhead with increased packet delivery ratio.
\end{abstract}

Keywords: VANET MAC, DIRECT.

\section{INTRODUCTION}

Vehicular ad hoc network (VANET) in contemporary ages has appeared as a gifted wireless network skill to upkeep a varied range of uses. VANET approves devoted short-range communication (DSRC) expertise which is centred on IEEE $802.11 p$ standard. It facilitates wireless communication among vehicles and between vehicles (V2V) and road side units (RSUs).

V2Vcommunication forms a basis for decentralized active safety applications that are expected to reduce accidents and their severity [1].

The applications of VANETs drop into two classes, viz. secured applications and non-secured applications. The secured applications have stringent necessities on communication dependability and suspension. Conversely, the non-secured applications are more throughput-sensitive rather than delay-sensitive [2].

The MAC procedure is accountable for distributing the communal medium amongst the competing nodules. The main objective of an effectual MAC procedure is to honestly segment communal sources like bandwidth, and access period amid partaking nodules, while also making the most of output and diminishing access suspensions.

The exclusive features of VANET enforce numerous restraints onto MAC procedure strategy. Significant objectives in scheming a MAC etiquette for VANET are justice, Quality of Service (QoS), dependability, time-bound distribution, sturdiness, dispersed, and on request network access, amongst others.

Revised Manuscript Received on December 13, 2019.

* Correspondence Author

M. Manoj., Scientist 'E' RCI, DRDO, Hyderabad

Email: man.obcd@gmail.com

A.CH. Sudhir, Asst. Professor, Dept. of Electronics and

Communication Engg .GITAM (Deemed to be University)

Email: sudhir.ach1@gmail.com
Because of the usage of a distinct radio channel, the MAC procedure is of supreme reputation in VANET schemes. [3]. The IEEE 802.11 p pile also services the typical CSMA/CA MAC which rapidly displays its incapability to put up augmented system traffic due to the absence of ACKs [4]. Out-dated wireless MAC procedures are unsuitable for VANETs that may effect in impacts amongst nodules, and much of the bandwidth of wireless network will be unexploited [5].

\section{RELATED WORKS}

Nishu Gupta et al [1] have suggested WAVE, which is an addition to IEEE $802.11 \mathrm{p}$ procedure. It goals listed distribution of security messages while concurrently facilitating the distribution of non-secured messages. It trusts on active group of inspirations to alleviate network cramming and incompetent bandwidth use by decreasing broadcast occurrence of inspirations.

Duc Ngoc Minh Dang et al [2] have proposed an Effectual and Dependable MAC procedure for VANETs (VER-MAC) that permits nodules to transmit security packages two times in the course of both the regulator network interim and facility network interim to upsurge the security transmission dependability. By expending the added data arrangements, nodules can convey facility packages in the course of the regulator network interim to increase the facility output.

Yi Cao et al [7] have proposed $\mathrm{CCH}$ architecture. The $\mathrm{CCH}$ architecture utilised to decrease the source ingestion of contention windows (CWs). The Suggested $\mathrm{CCH}$ construction cares similar disputation appliances and permits failures to alter their objective resource blocks (RBs) in the course of the altered MCBC. Moreover, to quicken the RB task, a cluster disputation policy is established by separating the whole structure into numerous clusters with diverse dimensions.

Ghassan Samara et al [8] have suggested a novel impact free procedure hereafter will be named (CF-MAC) to bring about the network access in Medium Access Control Sub-layer (MAC) for the broadcast will be suggested, that procedure will guarantee an impact-free administration to improve the network presentation, and upsurge the network dependability. The assessment norms will rely on the QoS improvement like network output, communication interruption, and communication harm.

\section{III.PROBLEM IDENTIFICATION}

In SAFE-MAC [3], the vehicles are grouped into batches based on their residence time and then assigned priorities. But in case of $\mathrm{V} 2 \mathrm{~V}$ communication, if the target vehicle is not reachable, then packets may not be transmitted correctly. Moreover due to the back off timer, the waiting time for the low priority vehicles will be higher. 
In OCA-MAC [5], cooperative communication is applied through some intermediate helper vehicles. But this protocol selects the cooperative nodes based on distance between the source and destination vehicles, without considering the residence time of the vehicles. Moreover, the optimum number of cooperative nodes is determined based on the throughput only, ignoring the packet error rate and packet delivery ratio at the receiver.

\section{MOBILITY AWARE COOPERATIVE MAC PROTOCOL (MAC-MAC)}

\section{A. Overview}

In order to solve the above identified problems, we propose to design a Mobility Aware Cooperative MAC protocol for successful transmission in VANET. In this protocol, when data is transmitted from $\mathrm{V} 2 \mathrm{~V}$, the packet error rate (PER) and packet delivery ratio (PDR) metrics are checked at the receiver end. Depending on these values, the transmission mode of the source is changed as DIRECT or COOPERATIVE. In cooperative mode, the source selects the potential relay nodes based on the residence time and distance to the receiver. A Fuzzy Decision model is then used to determine the required number of relay nodes based on the received PDR values and the relaying cost.

\section{B. System Model and Assumptions}

In this paper, we consider a modest VANET in which vehicles shift in a straight road with a connection with bidirectional congestion enclosed by a RSU, as exposed in Figure 1

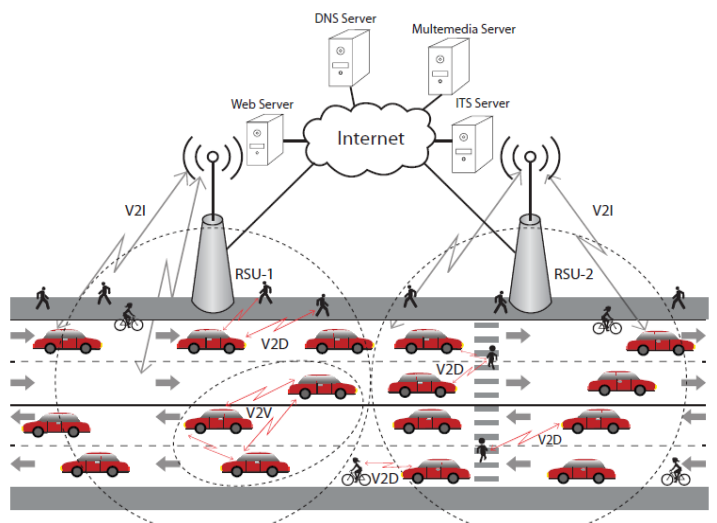

Figure 1 Architecture of VANET

The following assumptions are made in this paper:

- Vehicle to RSU relations are symmetric and the possessions of concealed stations, uncovered stations, and network detention are disregarded.

- Vehicles can calculate their poignant route, rapidity, and location surrounded by the system by means of a GPS receiver on their OBUs.

- Two non-overlapping networks are made use of by adjoining RSUs and these networks will not impede with one another.

- Vehicles are fortified with IEEE 802.11 empowered communication strategies.

- All vehicles and RSUs have certain exclusive documentation numeral.

- There occurs not less than single obtainable period slot, in order that the obliging nodule can communicate the records.

\section{a. Cooperative Condition}

Each node maintains its neighbour list by exchanging its control frame periodically. The format of the control frame is shown in Table 1.

Table -1 Format of Control frame

\begin{tabular}{|l|l|l|l|l|l|l|}
\hline Node & Assigned & \multicolumn{2}{|l|}{ Neighbour 1 } & \multicolumn{2}{l|}{ Neighbour2 } & $\ldots \ldots \ldots$. \\
\cline { 3 - 6 } Id & time slot & Id & $\begin{array}{l}\text { Assigned } \\
\text { time slot }\end{array}$ & Id & $\begin{array}{l}\text { Assigned } \\
\text { time slot }\end{array}$ & $\ldots \ldots$. \\
\hline
\end{tabular}

Let $\mathrm{S}$ and $\mathrm{D}$ be the source and destination vehicles respectively.

A cooperative or relay node is the common neighbour of $S$ and D. It can forward the data of S to D, repeatedly in free time slot.

Let be TR_MODE be the transmission mode of S which is initialized to DIRECT.

PDR is the ratio of correctly received packets by a receiver to the total number of packets sent. PER is the packet error rate measured at $\mathrm{D}$.

The steps involved in this process are illustrated below:

1. At D, PER and PDR are measured using Eq. (1) and (2), respectively

$$
\begin{aligned}
& \operatorname{PER}(\rho)=\frac{1}{1+\left(w_{n} \rho\right)^{v_{n}}} \\
& \forall \rho \geq 0 \\
& \operatorname{PDR}=\frac{N R(t)}{N S(t)}
\end{aligned}
$$

where, $\mathrm{w}_{\mathrm{n}}$ and $\mathrm{v}_{\mathrm{n}}$ are parameters that depends on AMC mode and packet size

$\rho$ Denotes the signal to noise-plus-interference ratio (SNIR)

$\mathrm{Nr}$ denotes the truncated ARQ AMC parameter

$\mathrm{NR}(\mathrm{t})$ is the number of correctly received packets by $\mathrm{D}$, at time $\mathrm{t}$

$\mathrm{NS}(\mathrm{t})$ is the total number of packets sent by $\mathrm{S}$, before $\mathrm{t}$.

2.If (PER $>\lambda$ and PDR $<\mu$ ), Then

D prepares RETRANSMIT: (Sid, PER, PDR) to

$\mathrm{S}$, where Sid is the Id of the source.

D broadcasts RETRANSMIT towards $\mathrm{S}$

Else If (PER $>\lambda$ and PDR $>\mu$ ), Then

D prepares RETRANSMIT: (Sid, PER) to $S$,

D broadcasts RETRANSMIT towards $S$

Else If (PER $<\lambda$ and PDR $<\mu$ ), Then

D prepares RETRANSMIT: (Sid, PDR) to $S$,

D broadcasts RETRANSMIT towards $S$

Else

D sends ACK towards $S$

End if

3. If $\mathrm{S}$ receives RETRANSMIT from $\mathrm{D}$, then Change TR_MODEto COOPERATIVE

End if

4.If TR_MODE $=$ COOPERATIVE

$\mathrm{S}$ invokes Relay Selection Algorithm

$S$ retransmits the packets using cooperative transmission End if 


\section{Relay Selection}

\section{a.Determination of Dwelling time}

When a vehicle enters into the service area of a RSU, the RSU estimates the dwelling time of that vehicle.

The dwelling time of a vehicle $\mathrm{N}$ at a specific RSU can be given by

$$
T_{D}(N)=\left\{\begin{array}{l}
\frac{R+r}{v}, \mid D=D+ \\
\frac{R-r}{v}, \mid D=D-
\end{array}\right.
$$

Where $\mathrm{R}$ is the radius of RSU

$r$ is the distance between $\mathrm{N}$ and RSU

$\mathrm{v}$ is the average velocity of $\mathrm{N}$

$\mathrm{D}$ is the moving direction of $\mathrm{N}$

$\mathrm{D}+$ indicates the direction towards RSU

D- indicates the direction away from RSU

\section{b. Relay selection Algorithm}

Let $\mathrm{nL}$ be the number of time slots allocated to a vehicle for transmission

Let $R_{1}$ be the radius assigned by $S$ for its cooperative transmission

Let $\mathrm{N}_{\mathrm{j}}$ be the vehicles within the radius of $\mathrm{R}_{\mathrm{l}}$

Let $d_{r}(j)$ be the distance between the vehicle $N_{j}$ and the destination.

1. If TR_MODE $=$ COOPERATIVE, Then

2. $S$ broadcasts the RELAY_REQ: $\left(\mathrm{S}_{\mathrm{id}}, \mathrm{D}_{\mathrm{id}}\right)$ to the vehicles within $R_{1}$.

3. If $\mathrm{N}_{\mathrm{j}}$ receives RELAY_REQ from $\mathrm{S}$, then

4. $\mathrm{N}_{\mathrm{j}}$ estimates $\mathrm{T}_{\mathrm{D}}\left(\mathrm{N}_{\mathrm{j}}\right)$ using (3)

5. $\mathrm{N}_{\mathrm{j}}$ estimates $\mathrm{d}_{\mathrm{r}}(\mathrm{j})$

6. $\quad \mathrm{N}_{\mathrm{j}}$ transmits a RELAY_REP: $\left(\mathrm{d}_{\mathrm{r}}(\mathrm{j}), \mathrm{T}_{\mathrm{D}}\left(\mathrm{N}_{\mathrm{j}}\right)\right)$ to $\mathrm{S}$

7. End if

8. If $\mathrm{S}$ receives RELAY_REP from $\mathrm{N}_{\mathrm{j}}$, then

9. If $\mathrm{d}_{\mathrm{r}}(\mathrm{j})=$ minimum and $\mathrm{T}_{\mathrm{D}}\left(\mathrm{N}_{\mathrm{j}}\right)=$ maximum , then

10. Select $\mathrm{N}_{\mathrm{j}}$ as relay node

11. Allocate time slot $\mathrm{nL}$ to $\mathrm{N}_{\mathrm{j}}$

12. $\mathrm{S}$ transmits data to $\mathrm{N}_{\mathrm{j}}$, cooperatively.

13. End if

14. End if

15. End if

In this algorithm, the source $S$ broadcast a relay request packet to the vehicles within a radius of $R_{1}$. On receiving this request, each potential nodes estimates the distance to the destination and its dwelling time. It then transmits a relay reply packet with the estimated values. On receiving the reply packet from all potential nodes, the source selects the node with minimum distance and maximum dwelling time as its candidate relay node. It then starts cooperative transmission to the selected relay node. The relay node is allocated the required number of free slots during which it forwards the packets to the destination.

\section{RESULT AND DISCUSSION}

\section{A. Simulation Parameters}

We use NS2 to simulate our proposed Mobility Aware Cooperative MAC (MACMAC) protocol. We compare our proposed protocol with OCAMAC protocol. The following metrics such as E2D, PDR, Drop, Overhead and Energy consumption are analyzed in our simulation.

Our simulation settings and parameters are summarized in table 1

Table 1: Simulation parameters

\begin{tabular}{|l|l|}
\hline No. of Nodes & 73 \\
\hline Area & $2500 \times 700$ \\
\hline MAC & 802.11 \\
\hline Simulation Time & $50 \mathrm{sec}$ \\
\hline Traffic Source & CBR \\
\hline Propagation & Two Ray Ground \\
\hline Antenna & Omni Antenna \\
\hline Initial Energy & $7.1 \mathrm{~J}$ \\
\hline Transmission Power & 0.3 \\
\hline Receiving Power & 0.3 \\
\hline Range & $250,300,350$ and $400 \mathrm{~m}$ \\
\hline Flows & $1,2,3,4,5$ and 6 \\
\hline
\end{tabular}

B. Based on Flows

In our first experiment we vary the number of flows as $1,2,3,4$ and 5 .

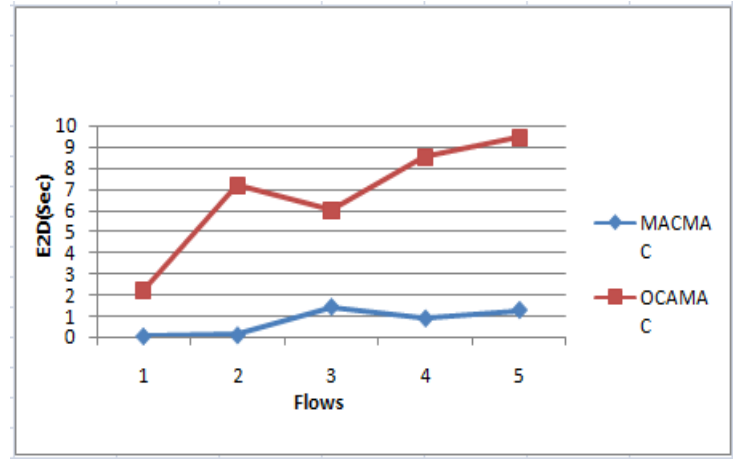

Fig 2: E2D for varying Flows

The result graph of E2D for different number of flows is shown in Figure 2. It can be observed that the E2D of MACMAC ranges from 0.03 to 1 seconds and the E2D of OCAMAC ranges from 2.2 to 9.4 seconds. Ultimately, the E2D of MACMAC has $90 \%$ lesser than OCAMAC.

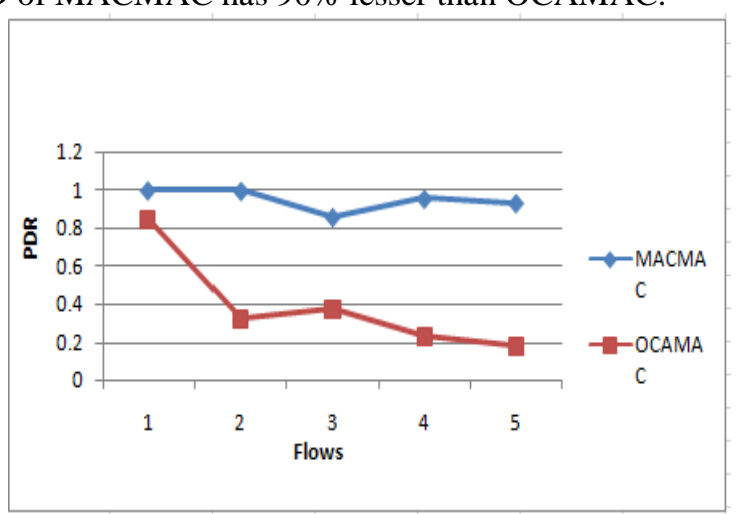

Fig 3: PDR for varying Flows

The result graph of PDR for different number of flows is shown in Figure 3. It can be observed that the PDR of MACMAC ranges from 0.99 to 0.93 and the PDR of OCAMAC ranges from 0.84 to 0.18 . Ultimately, the PDR of MACMAC has $59 \%$ higher than OCAMAC. 


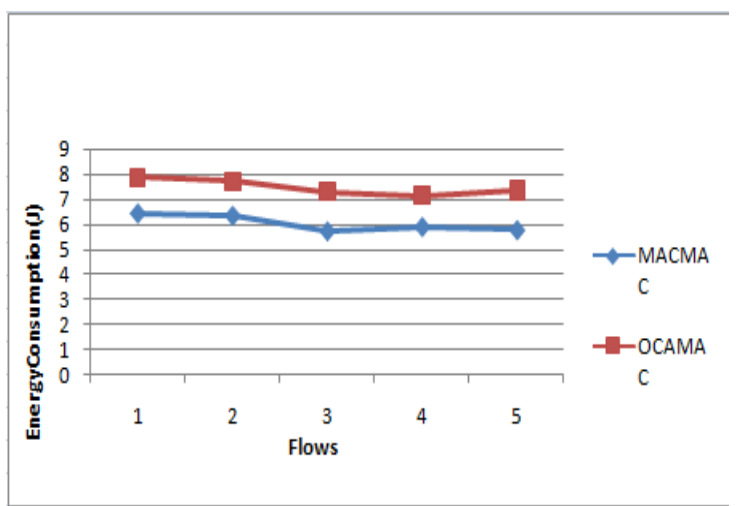

Fig 4: Energy consumption for varying Flows

The result graph of energy consumption for different number of flows is shown in Figure 4 . It can be observed that the energy consumption of MACMAC ranges from 6.4 to 5.7joules and the energy consumption of OCAMAC ranges from 7.8 to 7.3Joules Ultimately, the energy consumption of MACMAC has $19 \%$ lesser than OCAMAC.

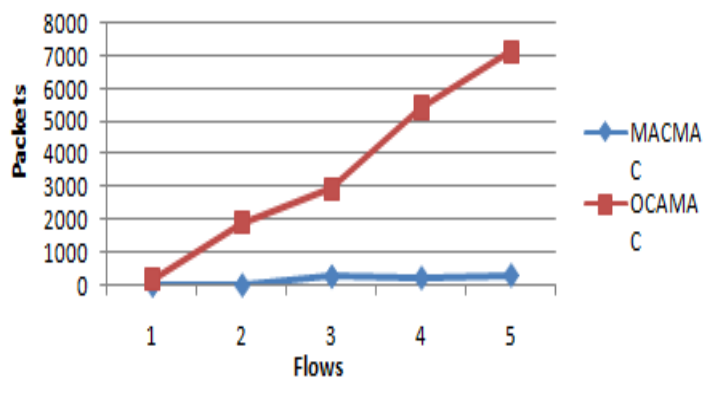

Fig 5: Packet Drop for varying Flows

The result graph of packet drop for different number of flows is shown in Figure 5. It can be observed that the drop of MACMAC ranges from 2 to 301 and the drop of OCAMAC ranges from 167 to 7131 . Ultimately, the drop of MACMAC has $96 \%$ lesser than OCAMAC.

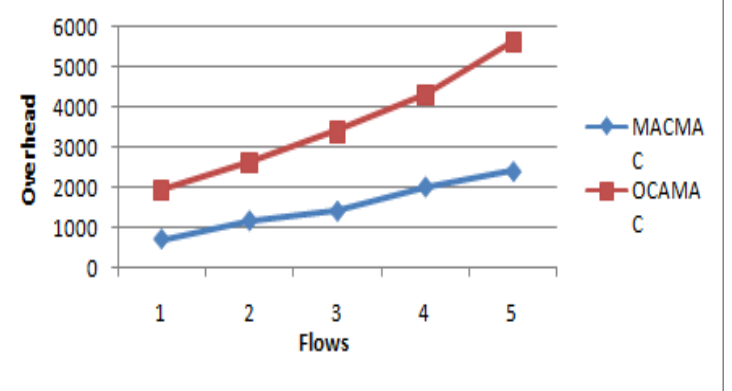

Fig 6: Overhead for varying Flows

The result graph of overhead for different number of flows is shown in Figure 6. It can be observed that the overhead of MACMAC ranges from 696 to 2408 and the overhead of OCAMAC ranges from 1948 to 5623. Ultimately, the overhead of MACMAC has $58 \%$ lesser than OCAMAC.

\section{Based on Range}

In our second experiment we vary the transmission range as $250,300,350$ and $400 \mathrm{~m}$.

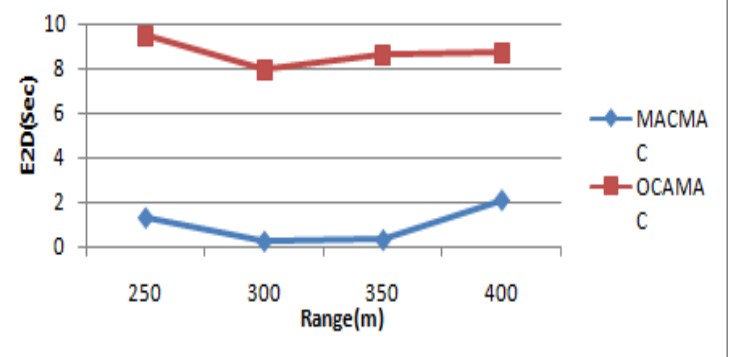

Fig 7: E2D for varying Range

The result graph of E2D for different range is shown in Figure 7. It can be observed that the E2D of MACMAC ranges from 12 to 2.0 seconds and E2D of OCAMAC ranges from 9.4 to 8.6 seconds. Ultimately, the E2D of MACMAC has $89 \%$ lesser than OCAMAC.

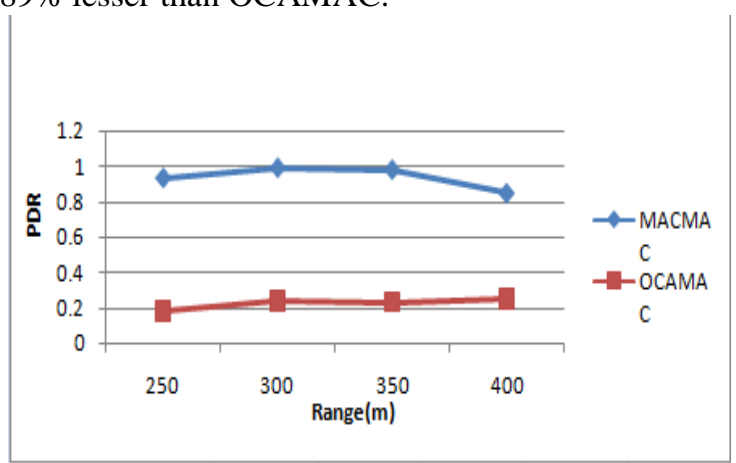

Fig 8: PDR for varying Range

The result graph of PDR for different range is shown in Figure 8. It can be observed that the PDR of MACMAC ranges from 0.93 to 0.84 and the PDR of OCAMAC ranges from 0.18 to 0.24 . Ultimately, the PDR of MACMAC has $76 \%$ higher than OCAMAC.

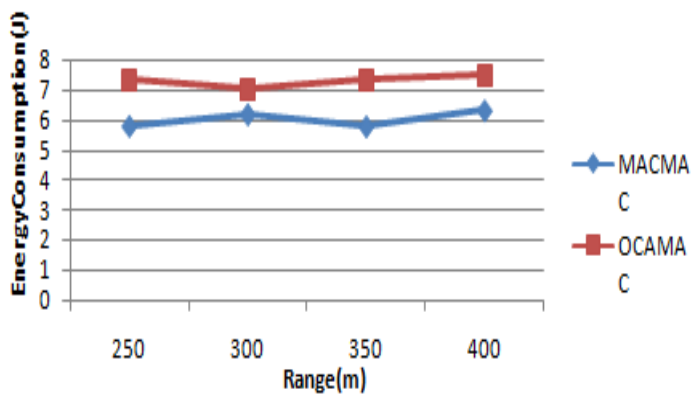

Fig 9: Energy consumption for varying Range The result graph of energy consumption for different range is shown in Figure 9. It can be observed that the energy consumption of MACMAC ranges from 5.7 to 6.3 joules and the energy consumption of OCAMAC ranges from 7.3 to 7.4 Joules Ultimately, the energy consumption of MACMAC has $18 \%$ lesser than OCAMAC. 


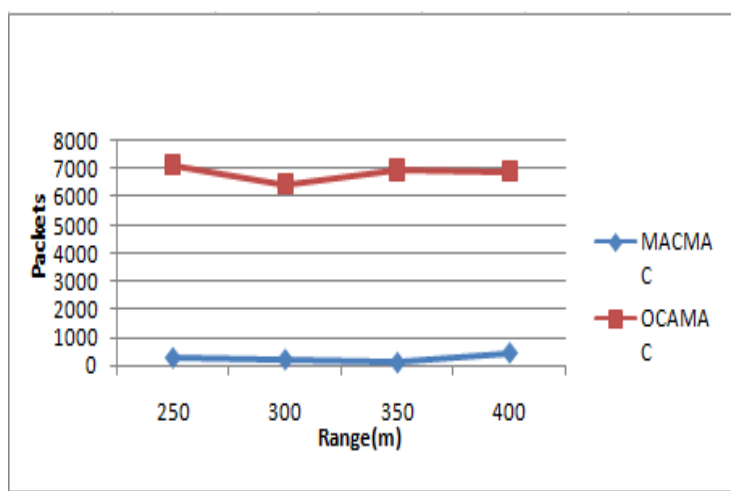

Fig 10: Packet Drop for varying Range

The result graph of packet drop for different range is shown in Figure 10. It can be observed that the drop of MACMAC ranges from 301 to 478 and the drop of OCAMAC ranges from 7131 to 6889. Ultimately, the drop of MACMAC has $96 \%$ lesser than OCAMAC.

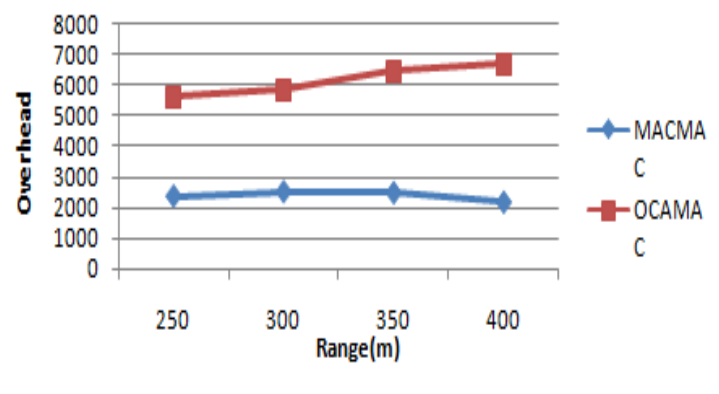

Fig 11: Overhead for varying Range

The result graph of overhead for different range is shown in Figure 11. It can be observed that the overhead of MACMAC ranges from 2408 to 2209 and the overhead of OCAMAC ranges from 5623 to 6702 . Ultimately, the overhead of MACMAC has $60 \%$ lesser than OCAMAC.

\section{CONCLUSION}

In this paper, Mobility Aware Cooperative MAC protocol (MAC-MAC) for successful transmission in VANET is proposed. In this protocol, when data is transmitted from $\mathrm{V} 2 \mathrm{~V}$, the packet error rate (PER) and packet delivery ratio (PDR) metrics are checked at the receiver end. Depending on these values, the transmission mode of the source is changed as DIRECT or COOPERATIVE. In cooperative mode, the source selects the potential relay nodes based on the residence time and distance to the receiver. By experimental results, it is shown that the proposed MACMAC protocol has reduced packet drops, delay and overhead with increased packet delivery ratio.

\section{REFERENCES}

1. Nishu Gupta, ArunPrakash, and Rajeev Tripathi, "Adaptive Beaconing in Mobility Aware Clustering Based MAC Protocol for Safety Message Dissemination in VANET", Wireless Communications and Mobile Computing, Volume 2017, Article ID 1246172, 15 pages, 2017.

2. Duc Ngoc Minh Dang,ChoongSeonHong,Sungwon Lee and EuiNam Huh, "An Efficient and Reliable MAC in VANETs",IEEE COMMUNICATIONS LETTERS, VOL. 18, NO. 4, APRIL 2014.

3. Md. AbubakarSiddik, ShafikaShowkatMoni, Mohammad Shah Alam and William A. Johnson, "SAFE-MAC: Speed Aware Fairness Enabled MAC Protocol for Vehicular Ad-hoc Networks",Sensors2019, 19, 2405; doi:10.3390/s19102405,2019.
4. Andreas Pressas, Zhengguo Sheng, Falah Ali, DaxinTian and MaziarNekovee, "Contention-based Learning MAC Protocol for Broadcast Vehicle-to-Vehicle Communication",IEEE,2018.

5. Yao Liu, Hongjing Zhou and Jiawei Huang, "OCA-MAC: A Cooperative TDMA-Based MAC Protocol for Vehicular Ad Hoc Networks",Sensors 2019, 19, 2691; doi:10.3390/s19122691,2019.

6. Md. KowsarHossain, SuprakashDatta, Sk. Imran Hossainand Jeff Edmonds, "ResVMAC: A Novel Medium Access Control Protocol for Vehicular Ad hoc Networks", Elsevier,Procedia Computer Science 109C (2017),pp:432-439,2017.

7. Yi Cao, HaixiaZhang,Dalei Wu and Dongfeng Yuan, "OGCMAC: A Novel OFDM Based Group Contention MAC for VANET Control Channel",IEEE TRANSACTIONS ON WIRELESS COMMUNICATIONS, VOL. 16, NO. 9, SEPTEMBER 2017.

8. Ghassan Samara, "An Efficient Collision Free Protocol for VANET",International Journal of Computer Applications (0975 8887), Volume 180 - No.16, February 2018.

\section{AUTHORS PROFILE}

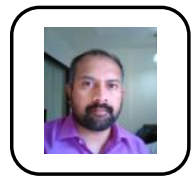

M.Manojreceived his B.Tech degree in Electronics and Communication Engineering from Osmania University, Hyderbad, M.Tech from Osmania University,Hyderabad in the field of Digital system engineering and pursuing Ph.D. in Dept. of ECE from GITAM deemed to be University, Visakhapatnam.

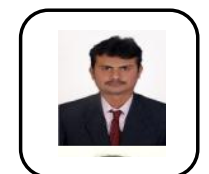

A.Ch.Sudhirreceived M.Tech degree in ECE from Andhra University, Visakhapatnam PGDES from University of Hyderabad ,MBA from Acharya Nagarjuna University and Ph.D in Wireless communication from JNTUK, kakinada. He has 14 years of teaching experience and presently working as Assistant Professor in Dept. ECE, GITAM deemed to be University, Visakhapatnam. He has almost 25 technical papers published in reputed journals and conferences. His research interests include Wireless communication and networks, Embedded systems, Optical fiber communication and Internet of things application messaging protocols. 